\title{
Coherent synchrobetatron resonance
}

\author{
A. Burov and V. Lebedev \\ Fermi National Accelerator Laboratory, Batavia, Illinois 60510, USA
}

(Received 16 April 2007; published 29 May 2007)

\begin{abstract}
Coherent synchrobetatron resonances can present a serious limit for low-energy synchrotrons. An excitation of a dipole transverse mode is considered at resonance condition. As an example, the growth rate for the Fermilab Booster is estimated.
\end{abstract}

DOI: 10.1103/PhysRevSTAB.10.054202

PACS numbers: 29.27.Eg

\section{INTRODUCTION}

Rapid cycling synchrotrons normally deal with so intense beams that the space charge tune shift can reach or even exceed 0.5. Therefore, to prevent integer resonances, the machine tunes have to be set just a little below integer numbers. Because of high acceleration rate, these machines have a rather high value of the synchrotron tune at injection energy. These circumstances can lead to an excitation of low-order coherent synchrobetatron resonance (CSBR). Coherent synchrobetatron resonances [1] have to be distinguished from single-particle ones [2]. Because of the high space charge tune shift, the coherent and incoherent spectra can be effectively separated, disabling Landau damping of that resonant mode (see, e.g., Ref. [3] and references therein). The resonant coherent mode is driven by an external source. The result is that its growth does not depend on the beam intensity, provided that the space charge is sufficient to switch off Landau damping. Presented calculations and some observations [4] indicate that CSBR is an important phenomenon for the Fermilab Booster; however, this paper is limited to the concept of CSBR, leaving related analysis of the observations for future reports.

\section{DIPOLE RESONANCES}

The condition of dipole CSBR is $\nu_{b}+l \nu_{s}=n$, with $\nu_{b}=\omega_{b} / \omega_{0}, \nu_{s}=\omega_{s} / \omega_{0}$ as the coherent betatron and synchrotron tunes, and $l, n$ as integer numbers. When this condition is satisfied, any dipole perturbation drives the resonant mode. CSBRs were first observed in simulations reported in Ref. [1]. It was pointed out there that they are driven by displacements and dispersion in rf cavities, although no analytical results were presented there. To understand the main features of the phenomenon, a longitudinal distribution is taken here as the air-bag one: all the particles have the same synchrotron amplitude $r_{0}$, and they are homogeneously distributed over the synchrotron phases $\phi$; the longitudinal offset is $z=r_{0} \cos \phi$. Following Ref. [5], the Vlasov equation for the distribution function $\psi$ can be written as

$$
\frac{\partial \psi}{\partial s}+\frac{\omega_{b}}{v_{0}} \frac{\partial \psi}{\partial \theta}+\frac{\omega_{s}}{v_{0}} \frac{\partial \psi}{\partial \phi}+\tilde{x}^{\prime} \frac{\partial \psi}{\partial x}+\tilde{p}_{x}^{\prime} \frac{\partial \psi}{\partial p_{x}}=0
$$

Here $s=v_{0} t$ is time in conventional units of length, $v_{0}$ is a longitudinal velocity, $x=q \cos \theta$ and $p_{x}=-\left(q / \beta_{x}\right) \sin \theta$ are the betatron coordinate and momentum (angle), $\tilde{x}^{\prime}=$ $d \tilde{x} / d s$ and $\tilde{p}_{x}^{\prime}=d \tilde{p}_{x} / d s$ are their perturbations. It can be assumed here that the perturbation kicks are localized at single point $s=s_{k}$; in case of many points the final result can be obtained by summation over them. Such localized perturbations are presented as

$$
\tilde{x}^{\prime}=\Delta x \delta_{P}\left(s-s_{k}\right) ; \quad \tilde{p}_{x}^{\prime}=\Delta p_{x} \delta_{P}\left(s-s_{k}\right),
$$

where

$$
\delta_{P}(s)=C^{-1} \sum_{m} \exp (-i m s / R)
$$

is the periodical delta function, $C=2 \pi R$ is the ring circumference.

For example, the perturbations may be caused by acceleration kicks in rf cavities, if there is some dispersion $D$ or its derivative $D^{\prime}$. In this case,

$$
\begin{aligned}
\Delta x & =-D \sin (k z) \Delta p_{\max } / p_{0} \\
\Delta p_{x} & =-\left(D^{\prime}+\frac{\alpha_{x}}{\beta_{x}} D\right) \sin (k z) \Delta p_{\max } / p_{0},
\end{aligned}
$$

where $k$ is the $\mathrm{rf}$ wave number, $\alpha_{x}$ and $\beta_{x}$ are the local Twiss parameters, $\Delta p_{\max }$ is an amplitude of the rf kick of the longitudinal momentum, and $p_{0}$ is the longitudinal momentum itself. Other possibilities for that sort of perturbation includes a mismatch between the dipole fields and momentum during acceleration (independent on the longitudinal position $z$ ), and dipole fields from image charges and currents (proportional to local linear density).

According to the conventional perturbation approach, a solution of the Vlasov Eq. (1) is presented as a sum of a steady state distribution and a perturbation: $\psi=\psi_{0}+\tilde{\psi}$. For the air-bag (hollow beam) distribution, 


$$
\begin{aligned}
\psi_{0}= & f_{0}(q) \delta\left(r-r_{0}\right) \\
\tilde{\psi}= & A(s) \sqrt{\beta_{x}} f_{0}^{\prime}(q) \delta\left(r-r_{0}\right) \\
& \times \exp \left(i \theta+i l \phi+i \chi z / r_{0}-i \Omega_{l} s / c\right) .
\end{aligned}
$$

Here $\Omega_{l}=\omega_{b}+l \omega_{s}=n \omega_{0}$ is a frequency of the considered resonance mode and $A(s)$ is its slowly growing amplitude, $f_{0}^{\prime}(q)=d f_{0} / d q$, and $\chi=\xi r_{0} /(R \eta)$ is the socalled head-tail phase, with the chromaticity $\xi=$ $d \nu_{b} / d\left(\Delta p / p_{0}\right)$ and the slippage factor $\eta$. Substituting Eqs. (5) in the Vlasov equation (1), neglecting the second-order terms $\propto \tilde{x}^{\prime} \tilde{\psi}, \tilde{p}_{x}^{\prime} \tilde{\psi}$ and leaving only a resonant contribution $m=n$ in the periodical delta-function expansion (3), a time derivative for the mode amplitude is obtained:

$$
\begin{aligned}
C \frac{d A}{d s}= & \left(\Delta p_{x} \sqrt{\beta_{x}} \sin \theta-\frac{\Delta x}{\sqrt{\beta_{x}}} \cos \theta\right) \\
& \times \exp \left(-i \theta-i l \phi-i \chi \cos \phi+i n s_{k} / R\right) .
\end{aligned}
$$

Averaging over the betatron phases, $e^{-i \theta} \sin \theta \rightarrow 1 /(2 i)$, $e^{-i \theta} \cos \theta \rightarrow 1 / 2$, averaging over the synchrotron phases, $\langle\cdots\rangle_{\phi}$, and summation over all the perturbations along the ring, $\sum_{k}$, leads to

$$
\begin{aligned}
C \frac{d A}{d s} & =\sum_{k} \frac{\exp \left(i n s_{k} / R\right)}{2}\langle\cdots\rangle_{\phi} ; \\
\langle\cdots\rangle_{\phi} \equiv & \left\langle\left(-i \Delta p_{x} \sqrt{\beta_{x}}-\frac{\Delta x}{\sqrt{\beta_{x}}}\right)\right. \\
& \times \exp (-i l \phi-i \chi \cos \phi)\rangle_{\phi} .
\end{aligned}
$$

For the dispersion-driven resonance, the kicks $\Delta x, \Delta p_{x}$ are given by Eqs. (4). Then, the term with averaging over the synchrotron phase is expressed by means of the Bessel functions:

$$
\begin{aligned}
\langle\cdots\rangle_{\phi}= & \frac{(-i)^{l+1}}{2 \sqrt{\beta_{x}}} \frac{\Delta p_{\max }}{p_{0}}\left[D+i\left(\beta_{x} D^{\prime}+\alpha_{x} D\right)\right] \\
& \times\left[J_{l}\left(\chi-k r_{0}\right)-J_{l}\left(\chi+k r_{0}\right)\right] .
\end{aligned}
$$

These equations give an increase per turn of the amplitude of the synchrobetatron dipole mode $l$ at the integer resonance $\Omega_{l}=n \omega_{0}$. In principle, this phenomenon is equivalent to excitation of a linear oscillator by an external resonance force.

In the vicinity of the resonance, $\Delta \Omega \equiv \Omega_{l}-n \omega_{0} \neq 0$, the right- hand side of Eq. (7) has to be multiplied by an oscillating factor $\exp (i \Delta \Omega s / c)$. If necessary, the Landau damping can be included adding $-\Lambda_{L} A$ in the right-hand side.
In principle, several ways can be foreseen to suppress CSBR: (i) to move the machine tune further from the integer, or put it in between the neighbor CSBRs; (ii) to increase the chromaticity, reducing the driving force in Eq. (7) and possibly introducing the Landau damping; (iii) in the opposite, to set the chromaticity so low that $\chi \approx$ 0 , so that the driving force for either even or odd modes will be zeroed, and to pick such a tune that the resonance mode is with a zero force; (iv) to cross CSBR faster; (v) to apply a 3rd harmonic in the rf, introducing more Landau damping.

Similarly, CSBR of any betatron order, $m \omega_{b}+l \omega_{s}=$ $n \omega_{0}, m \geq 2$, can be treated; however, it is not clear for the authors if the higher order CSBRs can be ever significant. Regarding the considered dipole CSBR, we estimated its possible influence for the Fermilab Booster at injection energy, using Eqs. (4) and (7); the growth can be as fast as $\simeq 30-60$ turns $/ \mathrm{mm}$. Some experimental details have been presented in Ref. [4]; their consideration from a point of view of CSBR is going to be a subject of a separate paper.

\section{CONCLUSION}

Analytical consideration of the coherent synchrobetatron resonance is presented for a simplified air-bag distribution. Essentially, the phenomenon results in a linear growth of the resonant coherent mode. This growth is driven by external field, so it is not sensitive to the beam intensity, as soon as Landau damping is switched off by the space charge. The phenomenon tends to be important for low-energy synchrotrons.

\section{ACKNOWLEDGMENTS}

We are thankful to W. Pellico and X. Yang for providing us with a stimulating measurement data, and to V. Danilov for exciting discussions.

[1] G. Besnier, D. Brandt, and B. Zotter, CERN/LEP-TH/8411, 1984.

[2] A. Piwinski, in Handbook of Accelerator Physics and Engineering, edited by A.W. Chao and M. Tigner (World Scientific, Singapore, 1999).

[3] O. Boine-Frankenheim, I. Hofmann, and V. Kornilov, in Proceedings of EPAC 2006.

[4] V. Lebedev, A. Burov, W. Pellico, and X. Yang, FERMILAB-CONF-06-205-AD, 2006.

[5] A. W. Chao, Physics of Collective Beam Instabilities in High Energy Accelerators (Wiley, New York, 1993). 